\title{
STABILITY AND OSCILLATION OF THE SOLUTIONS FOR A GENERALIZED COUPLED OSCILLATORS MODEL
}

\section{CHUNHUA FENG}

Department of Mathematics and Computer Science

Alabama State University

Montgomery, 36104

USA

e-mail: cfeng@alasu.edu

\begin{abstract}
In this paper, the stability and oscillation of the solutions for a class of generalized Hamiltonian parity-time non-symmetry model is investigated. By means of the mathematical analysis method, some sufficient conditions to guarantee the stability and oscillation of the solutions are obtained. Computer simulations are provided to demonstrate our results.
\end{abstract}

\section{Introduction}

It is known that a nonlinear system is a system in which the small change in input may produce an incommensurably large change in response. A coupled system of simple oscillators may often produce many new phenomena than isolated oscillator model. It gains much attention for engineers, physicists and mathematicians in last decade [1-15]. For 2010 Mathematics Subject Classification: 34K11.

Keywords and phrases: generalized coupled oscillators model, boundedness, stability, oscillation.

Received May 21, 2018; Revised July 23, 2018

(C) 2018 Scientific Advances Publishers 
example, Beregov and Melkikh have considered a system of autonomous inductively coupled Van der Pol generators, the mathematical model is the following:

$$
\left\{\begin{array}{l}
x_{1}^{\prime \prime}(t)-\left(\mu_{1}-x_{1}^{2}(t)\right) x_{1}^{\prime}(t)+x_{1}(t)=-M_{12} x_{2}^{\prime \prime}(t), \\
x_{2}^{\prime \prime}(t)-\left(\mu_{2}-x_{2}^{2}(t)\right) x_{2}^{\prime}(t)+x_{2}(t)=-M_{12} x_{1}^{\prime \prime}(t),
\end{array}\right.
$$

where $M_{12}$ is the coefficient of mutual induction which is either positive or negative number. The authors established the presence of a strange non-chaotic attractor and several stable limiting cycles [1]. Guin et al. have investigated a bilaterally coupled Rayleigh-Duffing oscillators model as follows:

$$
\left\{\begin{array}{l}
\left.x_{1}^{\prime \prime}(t)=a_{1} x_{1}(t)-b_{1} x_{1}^{3}(t)\right)+c_{1} x_{1}^{\prime}(t)-d_{1} x_{1}^{\prime 3}(t)+k_{1} f\left(x_{1}^{\prime}(t), x_{2}^{\prime}(t)\right), \\
\left.x_{2}^{\prime \prime}(t)=a_{2} x_{2}(t)-b_{2} x_{1}^{3}(t)\right)+c_{2} x_{2}^{\prime}(t)-d_{2} x_{2}^{\prime 3}(t)+k_{2} g\left(x_{1}^{\prime}(t), x_{2}^{\prime}(t)\right) .
\end{array}\right.
$$

With the increase of coupling factor between Rayleigh-Duffing oscillators, birth of periodic oscillations was observed. Dynamics becomes chaotic through a quasi-periodic route but for even higher coupling factor, synchronized stable periodic oscillations in Rayleigh-Duffing oscillators were found [2]. Tsoy has presented several models with parity-time symmetry. Hamiltonian functions for two and three linear oscillators coupled via coordinates and accelerations are derived. The mathematical model of two nonlinear oscillators is the following ([3], model (5), page 464):

$$
\left\{\begin{array}{l}
x_{1}^{\prime \prime}(t)+2 \gamma x_{1}^{\prime}(t)+w_{0}^{2} x_{1}(t)+k_{2} x_{2}(t)+\mu_{2} x_{2}^{\prime \prime}(t)=0, \\
x_{2}^{\prime \prime}(t)-2 \gamma x_{2}^{\prime}(t)+w_{0}^{2} x_{2}(t)+k_{1} x_{1}(t)+\mu_{1} x_{1}^{\prime \prime}(t)=0 .
\end{array}\right.
$$

Regions of stable dynamics for two coupled oscillators are obtained. Numerical solutions for model (3) are provided. The author pointed out that there is an infinite growth of coordinates and velocities in system (3) above the threshold. It means that the model under consideration is 
incomplete, so that more terms are necessary for an adequate description of the process. Then the author provided the following generalized Hamiltonian parity-time symmetry model:

$$
\left\{\begin{array}{l}
x_{1}^{\prime \prime}(t)+2 \gamma x_{1}^{\prime}(t)+w_{0}^{2} x_{1}(t)+k x_{2}(t)+\mu x_{2}^{\prime \prime}(t)+\left(x_{1}^{2}(t)+3 x_{2}^{2}(t)\right) x_{1}(t)=0, \\
x_{2}^{\prime \prime}(t)-2 \gamma x_{2}^{\prime}(t)+w_{0}^{2} x_{2}(t)+k x_{1}(t)+\mu x_{1}^{\prime \prime}(t)+\left(3 x_{1}^{2}(t)+x_{2}^{2}(t)\right) x_{2}(t)=0 .
\end{array}\right.
$$

However, the author did not discuss any dynamical properties for model (4). In other words, the dynamical property for system (4) is still an open problem. In this paper, we discuss the following general coupled oscillators model:

$$
\left\{\begin{array}{l}
x_{1}^{\prime \prime}(t)+\gamma_{1} x_{1}^{\prime}(t)+w_{1}^{2} x_{1}(t)+k_{2} x_{2}(t)+\mu_{2} x_{2}^{\prime \prime}(t)+\left(x_{1}^{2}(t)+3 x_{2}^{2}(t)\right) x_{1}(t)=0, \\
x_{2}^{\prime \prime}(t)-\gamma_{2} x_{2}^{\prime}(t)+w_{2}^{2} x_{2}(t)+k_{1} x_{1}(t)+\mu_{1} x_{1}^{\prime \prime}(t)+\left(3 x_{1}^{2}(t)+x_{2}^{2}(t)\right) x_{2}(t)=0,
\end{array}\right.
$$

where $\mu_{1} \mu_{2} \neq 1, \gamma_{i}, w_{i}^{2}, k_{i}, \mu_{i}(i=1,2)$ may be different numbers. By means of the mathematical analysis method, the boundedness, stability and oscillations of the solutions for model (5) are derived. It was emphasized that model (4) is a special case of system (5). Therefore, some dynamical behaviour of the generalized Hamiltonian parity-time symmetry model has been provided.

\section{Preliminaries}

System (5) can be written as the following:

$$
\left\{\begin{array}{l}
x_{1}^{\prime \prime}(t)=-\gamma_{1} x_{1}^{\prime}(t)-w_{1}^{2} x_{1}(t)-k_{2} x_{2}(t)-\mu_{2} x_{2}^{\prime \prime}(t)-\left(x_{1}^{2}(t)+3 x_{2}^{2}(t)\right) x_{1}(t), \\
x_{2}^{\prime \prime}(t)=\gamma_{2} x_{2}^{\prime}(t)-w_{2}^{2} x_{2}(t)-k_{1} x_{1}(t)-\mu_{1} x_{1}^{\prime \prime}(t)-\left(3 x_{1}^{2}(t)+x_{2}^{2}(t)\right) x_{2}(t),
\end{array}\right.
$$


or

$$
\left\{\begin{aligned}
x_{1}^{\prime \prime}(t)= & \frac{1}{1-\mu_{1} \mu_{2}}\left[-\gamma_{1} x_{1}^{\prime}(t)-w_{1}^{2} x_{1}(t)-k_{2} x_{2}(t)-\mu_{2} \gamma_{2} x_{2}^{\prime}(t)+\mu_{2} w_{2}^{2} x_{2}(t)\right. \\
& \left.+\mu_{2} k_{1} x_{1}(t)+\mu_{2}\left(3 x_{1}^{2}(t)+x_{2}^{2}(t)\right) x_{2}(t)-\left(x_{1}^{2}(t)+3 x_{2}^{2}(t)\right) x_{1}(t)\right], \\
x_{2}^{\prime \prime}(t)= & \frac{1}{1-\mu_{1} \mu_{2}}\left[\gamma_{2} x_{2}^{\prime}(t)-w_{2}^{2} x_{2}(t)-k_{1} x_{1}(t)+\mu_{1} \gamma_{1} x_{1}^{\prime}(t)+\mu_{1} w_{1}^{2} x_{1}(t)\right. \\
& \left.+\mu_{1} k_{2} x_{2}(t)+\mu_{1}\left(x_{1}^{2}(t)+3 x_{2}^{2}(t)\right) x_{1}(t)-\left(3 x_{1}^{2}(t)+x_{2}^{2}(t)\right) x_{2}(t)\right] .
\end{aligned}\right.
$$

For convenience, system (7) can be written as an equivalent four dimensional first order system:

$$
\left\{\begin{aligned}
x_{1}^{\prime}(t)= & \frac{1}{1-\mu_{1} \mu_{2}} x_{2}(t), \\
x_{2}^{\prime}(t)= & \left(\mu_{2} k_{1}-w_{1}^{2}\right) x_{1}(t)-\gamma_{1} x_{2}(t)+\left(\mu_{2} w_{2}^{2}-k_{2}\right) x_{3}(t)-\mu_{2} \gamma_{2} x_{4}(t) \\
& +\mu_{2}\left(3 x_{1}^{2}(t)+x_{3}^{2}(t)\right) x_{3}(t)-\left(x_{1}^{2}(t)+3 x_{3}^{2}(t)\right) x_{1}(t), \\
x_{3}^{\prime}(t)= & \frac{1}{1-\mu_{1} \mu_{2}} x_{4}(t), \\
x_{4}^{\prime}(t)= & \left(\mu_{1} w_{1}^{2}-k_{1}\right) x_{1}(t)+\mu_{1} \gamma_{1} x_{2}(t)+\left(\mu_{1} k_{2}-w_{2}^{2}\right) x_{3}(t)+\gamma_{2} x_{4}(t) \\
& +\mu_{1}\left(x_{1}^{2}(t)+3 x_{3}^{2}(t)\right) x_{1}(t)-\left(3 x_{1}^{2}(t)+x_{3}^{2}(t)\right) x_{3}(t) .
\end{aligned}\right.
$$

The system (8) can be expressed in the following matrix form:

$$
x^{\prime}(t)=A x(t)+f(x(t)),
$$


where $x(t)=\left(x_{1}(t), x_{2}(t), x_{3}(t), x_{4}(t)\right)^{T}, A$ is a 4 by 4 matrix, and $f(x)$ is a 4 by 1 vector:

$$
\begin{gathered}
A=\left(a_{i j}\right)_{4 \times 4}=\left(\begin{array}{cccc}
0 & \frac{1}{1-\mu_{1} \mu_{2}} & 0 & 0 \\
\mu_{2} k_{1}-w_{1}^{2} & -\gamma_{1} & \mu_{2} w_{2}^{2}-k_{2} & -\mu_{2} \gamma_{2} \\
0 & 0 & 0 & \frac{1}{1-\mu_{1} \mu_{2}} \\
\mu_{1} w_{1}^{2}-k_{1} & \mu_{1} \gamma_{1} & \mu_{1} k_{2}-w_{2}^{2} & \gamma_{2}
\end{array}\right), \\
f(x)=\left(\begin{array}{l}
f_{1}(x) \\
f_{2}(x) \\
f_{3}(x) \\
f_{4}(x)
\end{array}\right)=\left(\begin{array}{ccc}
\mu_{2}\left(3 x_{1}^{2}(t)+x_{3}^{2}(t)\right) x_{3}(t)-\left(x_{1}^{2}(t)+3 x_{3}^{2}(t)\right) x_{1}(t) \\
\mu_{1}\left(x_{1}^{2}(t)+3 x_{3}^{2}(t)\right) x_{1}(t)-\left(3 x_{1}^{2}(t)+x_{3}^{2}(t)\right) x_{3}(t)
\end{array}\right) .
\end{gathered}
$$

The linearized system of (9) is

$$
x^{\prime}(t)=A x(t)
$$

Obviously, system (9) can be seen as a distributed system of system (10). The distributed term is $f(x)$.

Lemma 1. Assume that the following inequality holds:

$$
\left(\mu_{2} k_{1}-w_{1}^{2}\right)\left(\mu_{1} k_{2}-w_{2}^{2}\right) \neq\left(\mu_{2} w_{2}^{2}-k_{2}\right)\left(\mu_{1} w_{1}^{2}-k_{1}\right),
$$

then there exists a unique equilibrium point which is exactly the zero point for system (8) (or (9)). 
Proof. An equilibrium point $x^{*}=\left[x_{1}^{*}, x_{2}^{*}, x_{3}^{*}, x_{4}^{*}\right]^{T}$ of system (8) is a constant solution of the following system:

$$
\left\{\begin{array}{l}
\frac{1}{1-\mu_{1} \mu_{2}} x_{2}^{*}=0, \\
\left(\mu_{2} k_{1}-w_{1}^{2}\right) x_{1}^{*}-\gamma_{1} x_{2}^{*}+\left(\mu_{2} w_{2}^{2}-k_{2}\right) x_{3}^{*}-\mu_{2} \gamma_{2} x_{4}^{*} \\
\quad+\mu_{2}\left[3\left(x_{1}^{*}\right)^{2}+\left(x_{3}^{*}\right)^{2}\right] x_{3}^{*}-\left[\left(x_{1}^{*}\right)^{2}+3\left(x_{3}^{*}\right)^{2}\right] x_{1}^{*}=0, \\
\frac{1}{1-\mu_{1} \mu_{2}} x_{4}^{*}=0, \\
\left(\mu_{1} w_{1}^{2}-k_{1}\right) x_{1}^{*}+\mu_{1} \gamma_{1} x_{2}^{*}+\left(\mu_{1} k_{2}-w_{2}^{2}\right) x_{3}^{*}+\gamma_{2} x_{4}^{*} \\
\quad+\mu_{1}\left[\left(x_{1}^{*}\right)^{2}+3\left(x_{3}^{*}\right)^{2}\right] x_{1}^{*}-\left[3\left(x_{1}^{*}\right)^{2}+\left(x_{3}^{*}\right)^{2}\right] x_{3}^{*}=0 .
\end{array}\right.
$$

Noting that $x_{2}^{*}=0, x_{4}^{*}=0$, so system (12) changes to the following:

$$
\left\{\begin{aligned}
\left(\mu_{2} k_{1}-\right. & \left.w_{1}^{2}\right) x_{1}^{*}+\left(\mu_{2} w_{2}^{2}-k_{2}\right) x_{3}^{*} \\
& +\mu_{2}\left[3\left(x_{1}^{*}\right)^{2}+\left(x_{3}^{*}\right)^{2}\right] x_{3}^{*}-\left[\left(x_{1}^{*}\right)^{2}+3\left(x_{3}^{*}\right)^{2}\right] x_{1}^{*}=0 \\
\left(\mu_{1} w_{1}^{2}-k_{1}\right) x_{1}^{*}+\left(\mu_{1} k_{2}-w_{2}^{2}\right) x_{3}^{*} & \\
& +\mu_{1}\left[\left(x_{1}^{*}\right)^{2}+3\left(x_{3}^{*}\right)^{2}\right] x_{1}^{*}-\left[3\left(x_{1}^{*}\right)^{2}+\left(x_{3}^{*}\right)^{2}\right] x_{3}^{*}=0 .
\end{aligned}\right.
$$

We shall prove that $x_{1}^{*}=0, x_{3}^{*}=0$. Indeed, system (13) can be written as a matrix form:

$$
B x^{*}=\mathbf{0},
$$

where $x^{*}=\left[x_{1}^{*}, x_{3}^{*}\right]^{T}, B$ is a 2 by 2 matrix:

$$
B=\left(\begin{array}{ll}
\mu_{2} k_{1}-w_{1}^{2}-\left[\left(x_{1}^{*}\right)^{2}+3\left(x_{3}^{*}\right)^{2}\right] & \mu_{2} w_{2}^{2}-k_{2}+\mu_{2}\left[3\left(x_{1}^{*}\right)^{2}+\left(x_{3}^{*}\right)^{2}\right] \\
\mu_{1} w_{1}^{2}-k_{1}+\mu_{1}\left[\left(x_{1}^{*}\right)^{2}+3\left(x_{3}^{*}\right)^{2}\right] & \mu_{1} k_{2}-w_{2}^{2}-\left[3\left(x_{1}^{*}\right)^{2}+\left(x_{3}^{*}\right)^{2}\right]
\end{array}\right) .
$$


Firstly, suppose that for any $x_{1}^{*}, x_{3}^{*}$, matrix $B$ is a nonsingular matrix. In other words, the following inequality holds:

$$
\begin{gathered}
\left\{\mu_{2} k_{1}-w_{1}^{2}-\left[\left(x_{1}^{*}\right)^{2}+3\left(x_{3}^{*}\right)^{2}\right]\right\}\left\{\mu_{1} k_{2}-w_{2}^{2}-\left[3\left(x_{1}^{*}\right)^{2}+\left(x_{3}^{*}\right)^{2}\right]\right\} \neq \\
\left\{\mu_{2} w_{2}^{2}-k_{2}+\mu_{2}\left[3\left(x_{1}^{*}\right)^{2}+\left(x_{3}^{*}\right)^{2}\right]\right\}\left\{\mu_{1} w_{1}^{2}-k_{1}+\mu_{1}\left[\left(x_{1}^{*}\right)^{2}+3\left(x_{3}^{*}\right)^{2}\right]\right\}
\end{gathered}
$$

then based on the linear algebraic Cramer's rule, system (14) only has trivial solution, namely, $x_{1}^{*}=0, x_{3}^{*}=0$. However, when $x_{1}^{*}=0, x_{3}^{*}=0$. Equation (15) changes to (11). The proof is completed.

Lemma 2. Assume that $\mu_{1}<0, \mu_{2}<0$, then the solutions of system (8) (or (9)) are bounded.

Proof. To prove the boundedness of the solutions in system (8), we construct a Lyapunov function $V(t)=\sum_{i=1}^{4} \frac{1}{2} x_{i}^{2}(t)$. Calculating the derivative of $V(t)$ through system (8), we have

$$
\begin{aligned}
& \left.V^{\prime}(t)\right|_{(8)}=\sum_{i=1}^{4} x_{i}(t) x_{i}^{\prime}(t) \\
& =\frac{1}{1-\mu_{1} \mu_{2}} x_{1}(t) x_{2}(t)+x_{2}(t)\left[\left(\mu_{2} k_{1}-w_{1}^{2}\right) x_{1}(t)-\gamma_{1} x_{2}(t)+\left(\mu_{2} w_{2}^{2}-k_{2}\right) x_{3}(t)\right. \\
& \left.\quad-\mu_{2} \gamma_{2} x_{4}(t)+\mu_{2}\left(3 x_{1}^{2}(t)+x_{3}^{2}(t)\right) x_{3}(t)-\left(x_{1}^{2}(t)+3 x_{3}^{2}(t)\right) x_{1}(t)\right] \\
& \quad+\frac{1}{1-\mu_{1} \mu_{2}} x_{3}(t) x_{4}(t)+x_{4}(t)\left[\left(\mu_{1} w_{1}^{2}-k_{1}\right) x_{1}(t)+\mu_{1} \gamma_{1} x_{2}(t)\right. \\
& \quad+\left(\mu_{1} k_{2}-w_{2}^{2}\right) x_{3}(t)+\gamma_{2} x_{4}(t)+\mu_{1}\left(x_{1}^{2}(t)\right. \\
& \left.\left.\quad+3 x_{3}^{2}(t)\right) x_{1}(t)-\left(3 x_{1}^{2}(t)+x_{3}^{2}(t)\right) x_{3}(t)\right]
\end{aligned}
$$




$$
\begin{gathered}
=\left[\frac{1}{1-\mu_{1} \mu_{2}}+\mu_{2} k_{1}-w_{1}^{2}\right] x_{1}(t) x_{2}(t)-\gamma_{1} x_{2}^{2}(t)+\left(\mu_{2} w_{2}^{2}-k_{2}\right) x_{2}(t) x_{3}(t) \\
-\mu_{2} \gamma_{2} x_{2}(t) x_{4}(t)+\left[\frac{1}{1-\mu_{1} \mu_{2}}+\mu_{1} k_{2}-w_{2}^{2}\right] x_{3}(t) x_{4}(t)+\left(\mu_{1} w_{1}^{2}-k_{1}\right) x_{1}(t) x_{4}(t) \\
\quad+\gamma_{2} x_{4}^{2}(t)+\mu_{2}\left(3 x_{1}^{2}(t)+x_{3}^{2}(t)\right) x_{2}(t) x_{3}(t)-\left(x_{1}^{2}(t)+3 x_{3}^{2}(t)\right) x_{1}(t) x_{2}(t) \\
\quad+\mu_{1}\left(x_{1}^{2}(t)+3 x_{3}^{2}(t)\right) x_{1}(t) x_{4}(t)-\left(3 x_{1}^{2}(t)+x_{3}^{2}(t)\right) x_{3}(t) x_{4}(t) .
\end{gathered}
$$

Noting that as $x_{i}(t) \rightarrow+\infty(i=1, \cdots, 4),\left(3 x_{1}^{2}(t)+x_{3}^{2}(t)\right) x_{2}(t) x_{3}(t),\left(x_{1}^{2}(t)+\right.$ $\left.3 x_{3}^{2}(t)\right) x_{1}(t) x_{2}(t),\left(x_{1}^{2}(t)+3 x_{3}^{2}(t)\right) x_{1}(t) x_{4}(t), \quad$ and $\quad\left(3 x_{1}^{2}(t)+x_{3}^{2}(t)\right) x_{3}(t) x_{4}(t)$ are higher order infinity than $x_{i}(t) x_{j}(t)(i, j=1, \cdots, 4)$. Therefore, when $\mu_{1}<0, \mu_{2}<0$, there exists $M>0$ such that $\left.V^{\prime}(t)\right|_{(8)}<0$ as $\left|x_{i}\right|>M$, $(i=1, \cdots, 4)$. This means that the all solutions of system (8) are bounded.

\section{Stability of the Solutions}

Theorem 1. Assume that all solutions of system (8) (or (9)) are bounded. If zero is the unique equilibrium point of system (8) (or (9)) for selecting parameter values. Let $\alpha_{1}, \alpha_{2}, \alpha_{3}, \alpha_{4}$ be characteristic values of matrix A. If $\alpha_{i}<0$, or $\operatorname{Re}\left(\alpha_{i}\right)<0,(i=1, \cdots, 4)$, then the trivial solution is stable.

Proof. Let $v_{i}=\left[v_{i 1}, \cdots, v_{i 4}\right]^{T}$ be the corresponding characteristic vectors of $\alpha_{i}(i=1,2,3,4)$. Then the solution to the linearized system (10) is the following:

$$
x_{i}(t)=c_{1} \nu_{1 i} \exp \left(\alpha_{1} t\right)+c_{2} \nu_{2 i} \exp \left(\alpha_{2} t\right)+c_{3} \nu_{3 i} \exp \left(\alpha_{3} t\right)+c_{4} \nu_{4 i} \exp \left(\alpha_{4} t\right),
$$

where $c_{i}(i=1,2,3,4)$ are any constants. Since $\alpha_{i}<0$ or $\operatorname{Re}\left(\alpha_{i}\right)<0$, $(i=1, \cdots, 4)$, this means that the trivial solution of system (10) is stable. 
Noting that $f_{1}(x)=f_{3}(x)=0$ in system (9). Both $f_{2}(x)$ and $f_{4}(x)$ are higher infinitesimal as $x_{1}(t) \rightarrow 0$ and $x_{3}(t) \rightarrow 0$. Hence, the stability of the trivial solution of system (10) implies the stability of the trivial solution of system (9).

\section{Oscillatory Behaviour of the Solutions}

Theorem 2. Assume that all solutions of system (9) are bounded. If zero is the unique equilibrium point of system (9) for selecting parameter values. Let $\alpha_{1}, \alpha_{2}, \alpha_{3}, \alpha_{4}$ be characteristic values of matrix A. If there exists some positive $\alpha_{k}$, or $\operatorname{Re}\left(\alpha_{k}\right) \geq 0,(k \in\{1,2,3,4\})$, then the unique equilibrium point of system (9) is unstable. System (9) (thus system (5)) generates an oscillatory solution.

Proof. Obviously, the trivial solution of system (9) is unstable if and only if the trivial solution of system (10) is unstable. Therefore, we only need to prove the instability of the trivial solution of system (10). Since $\alpha_{i}(i=1,2,3,4)$ are characteristic values of matrix $A$, then the characteristic equation corresponding to system (10) is the following:

$$
\prod_{i=1}^{4}\left(\lambda-\alpha_{i}\right)=0
$$

Without loss of generality, we assume that $\alpha_{1}>0$, or $\operatorname{Re}\left(\alpha_{1}\right) \geq 0$. Then from (18) we have

$$
\lambda-\alpha_{1}=0 .
$$

Since $\alpha_{1}>0$, or $\operatorname{Re}\left(\alpha_{1}\right)>0$, this means that there is a positive (or a positive real part) characteristic value of system (10). Therefore, the trivial solution of system (10) is unstable, implies that the trivial solution of system (9) is unstable. If $\operatorname{Re}\left(\alpha_{1}\right)=0$, this means that system (10) has a pure imaginary root. Since both $\sin t$ and $\cos t$ can not tend to zero as $t$ tends to infinity. Therefore, the trivial solution is unstable. The boundedness of the solutions of system (9) and the instability of unique equilibrium point will force system (9) to generate an oscillatory solution. 
Theorem 3. Assume that all solutions of system (9) are bounded. If zero is the unique equilibrium point of system (9) for selecting parameter values. Let $\mu(A)=\max _{1 \leq j \leq 4}\left[a_{j j}+\sum_{i=1, i \neq j}^{4}\left|a_{i j}\right|\right]$ [16]. If $\mu(A)>0$, then system (9) has an oscillatory solution.

Proof. Let $y(t)=\sum_{i=1}^{4}\left|x_{i}(t)\right|$, from (10), we have

$$
y^{\prime}(t) \leq \mu(A) y(t) .
$$

Consider the scalar differential equation

$$
z^{\prime}(t)=\mu(A) z(t)
$$

According to the comparison theorem of differential equation, we have $y(t) \leq z(t)$. For Equation (21), the characteristic equation associated with (21) is given by

$$
\lambda=\mu(A)
$$

Since $\mu(A)>0$, this means that there exists a positive characteristic root of Equation (21). Thus, the trivial solution of Equation (21) is unstable, implying that the trivial solution of Equation (10) is unstable. It suggested that system (9) (thus system (5)) has an oscillatory solution.

\section{Simulation Results}

The simulation is based on the equivalent system (8) of (5), first the parameters are selected as follows: $\mu_{1}=-0.45, \mu_{2}=-0.55, \gamma_{1}=0.15$, $\gamma_{2}=-0.25, k_{1}=0.08, k_{2}=0.16, w_{1}=0.25, w_{2}=0.45$, then the characteristic values of $A$ are $-0.1557 \pm 0.6937 i,-0.0001,-0.0887$. Based on Theorem 1, the trivial solution is convergent (see Figure 1). When the parameters are selected as $\mu_{1}=-0.35, \mu_{2}=-0.28, \gamma_{1}=-0.12$, $\gamma_{2}=-0.115, k_{1}=0.08, k_{2}=-0.06, w_{1}=0.25, w_{2}=0.15$, then the characteristic values of $A$ are $0.1514 \pm 0.2681 i,-0.1489 \pm 0.2381 i$. The 
conditions of Lemma 1 and Lemma 2 are satisfied. Since there is a positive real part of characteristic value $0.1514+0.2681 i$ of matrix $A$, based on Theorem 2, there exists an oscillatory solution for system (8) (see Figure 2). When the parameters are selected as $\mu_{1}=-0.48, \mu_{2}=-0.36, \gamma_{1}=0.15$, $\gamma_{2}=-0.25, k_{1}=-1.18, k_{2}=0.25, w_{1}=0.65, w_{2}=0.45$, then $\mu(A)=1.0489$. The conditions of Lemma 1 and Lemma 2 are satisfied. Since $\mu(A)=1.0489>0$, based on Theorem 3 , there exists an oscillatory solution for system (8) (see Figure 3).

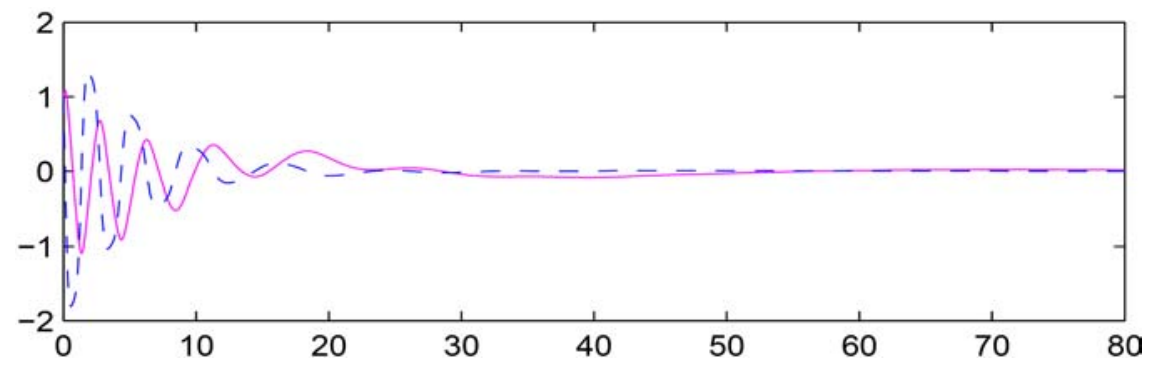

(a) Solid line: $x_{1}(t)$, dashed line: $x_{2}(t)$

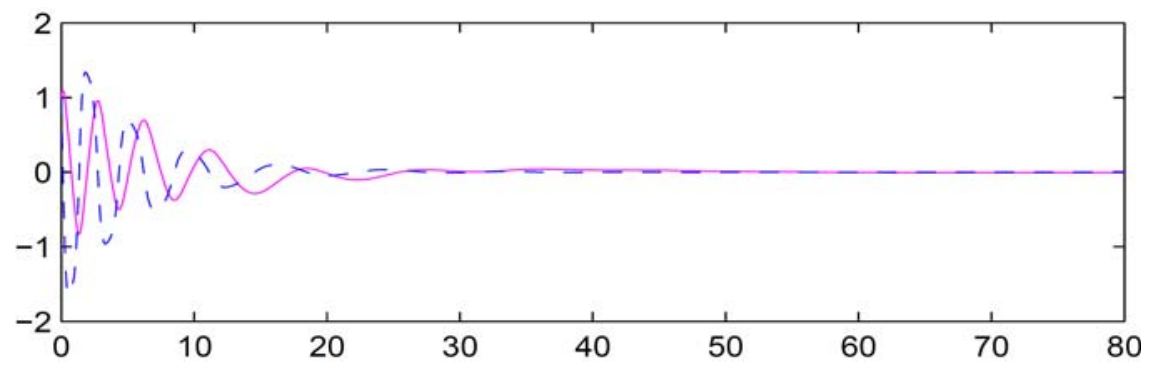

(b) Solid line: $x_{3}(t)$, dashed line: $x_{4}(t)$

Figure 1. The trivial solution is convergent. 


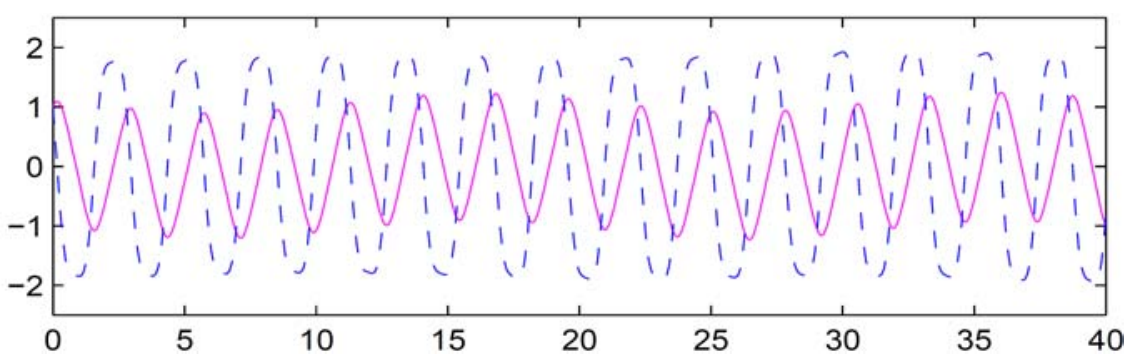

(a) Solid line: $x_{1}(t)$, dashed line: $x_{2}(t)$

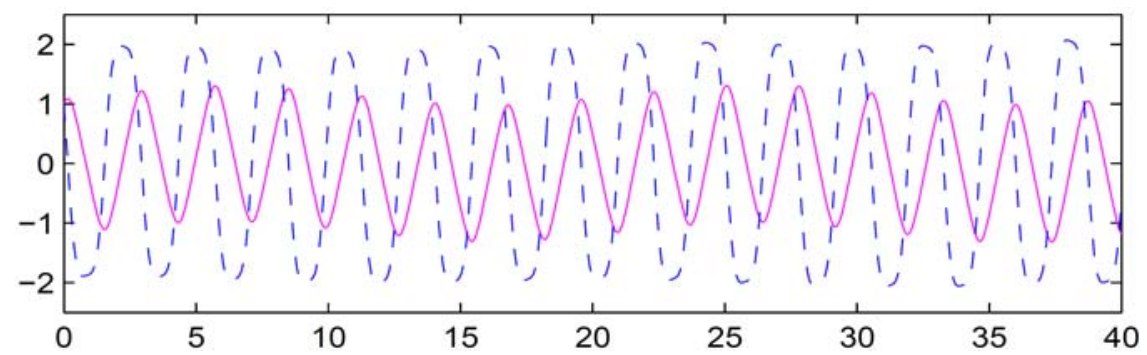

(b) Solid line: $x_{3}(t)$, dashed line: $x_{4}(t)$

Figure 2. Oscillatory behaviour of the solutions. 


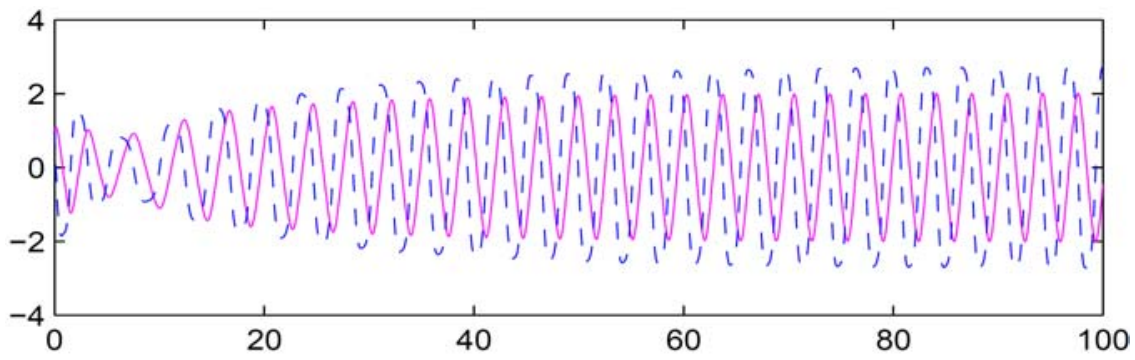

(a) Solid line: $x_{1}(t)$, dashed line: $x_{2}(t)$

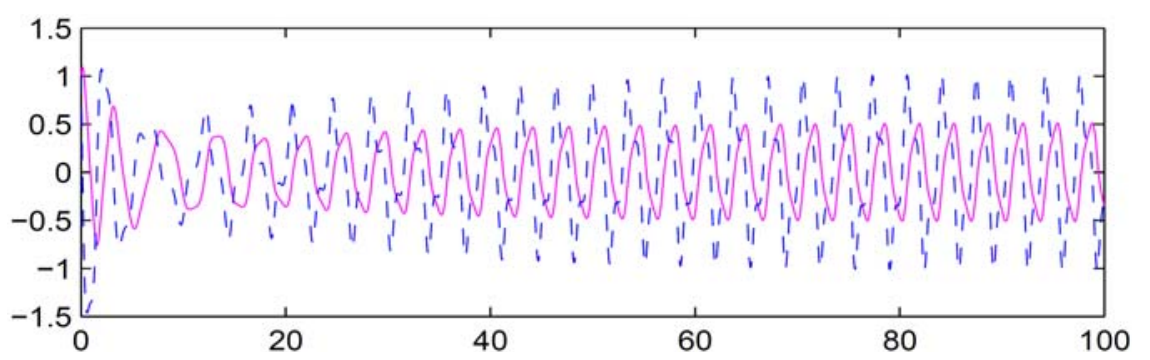

(b) Solid line: $x_{3}(t)$, dashed line: $x_{4}(t)$

Figure 3. Oscillatory behaviour of the solutions.

\section{Conclusion}

In this paper, we have discussed the convergence and oscillatory behaviour of the solutions for a generalized coupled oscillators model. Based on mathematical analysis method, we provided some sufficient conditions to guarantee the stability and oscillation of the solutions. Some simulations are provided to indicate the effectness of the criterion.

\section{References}

[1] R. Y. Beregov and A. V. Melkikh, De-synchronization and chaos in two inductively coupled Van der Pol auto-generators, Chaos, Solitons and Fractals 73 (2015), 17-28.

DOI: https://doi.org/10.1016/j.chaos.2014.12.017

[2] A. Guin, M. Dandapathak, S. Sarkar and B. C. Sarkar, Birth of oscillation in coupled non-oscillatory Rayleigh-Duffing oscillators, Communications in Nonlinear Science and Numerical Simulation 42 (2017), 420-436.

DOI: https://doi.org/10.1016/j.cnsns.2016.06.002 
[3] E. N. Tsoy, Coupled oscillators with parity-time symmetry, Physics Letters A 381(5) (2017), 462-466.

DOI: https://doi.org/10.1016/j.physleta.2016.12.023

[4] P. F. Xu and Y. F. Jin, Stochastic resonance in multi-stable coupled systems driven by two driving signals, Physica A: Statistical Mechanics and its Applications 492 (2018), 1281-1289.

DOI: https://doi.org/10.1016/j.physa.2017.11.056

[5] V. Settimi and F. Romeo, Dynamic regimes of a nonlinearly coupled electromechanical system, International Journal of Non-Linear Mechanics 103 (2018), 68-81.

DOI: https://doi.org/10.1016/j.ijnonlinmec.2018.04.008

[6] J. J. Gil, P. Ciaurriz and I. Diaz, Controlling two haptically-coupled devices: System modeling and stability analysis, Mechatronics 49 (2018), 224-234.

DOI: https://doi.org/10.1016/j.mechatronics.2017.12.010

[7] A. Neirameh, M. Eslami and S. Shokooh, New solution algorithm of coupled nonlinear system of Schrodinger equations, Alexandria Engineering Journal 57(1) (2018), 247-253.

DOI: https://doi.org/10.1016/j.aej.2016.12.003

[8] M. O. Fen, Persistence of chaos in coupled Lorenz systems, Chaos, Solitons and Fractals 95 (2017), 200-205.

DOI: https://doi.org/10.1016/j.chaos.2016.12.017

[9] J. C. Ji and J. Zhou, Coexistence of two families of sub-harmonic resonances in a time-delayed nonlinear system at different forcing frequencies, Mechanical Systems and Signal Processing 93 (2017), 151-163.

DOI: https://doi.org/10.1016/j.ymssp.2017.02.007

[10] H. Fotouhi, S. Moryadee and E. M. Hooks, Quantifying the resilience of an urban traffic-electric power coupled system, Reliability Engineering and System Safety 163 (2017), 79-94.

DOI: https://doi.org/10.1016/j.ress.2017.01.026

[11] S. Aljoudi, B. Ahmad, J. J. Nieto and A. Alsaedi, A coupled system of Hadamard type sequential fractional differential equations with coupled strip conditions, Chaos, Solitons and Fractals 91 (2016), 39-46.

DOI: https://doi.org/10.1016/j.chaos.2016.05.005

[12] P. Brzeski, E. Pavlovskaia, T. Kapitaniak and P. Perlikowski, Controlling multistability in coupled systems with soft impacts, International Journal of Mechanical Sciences 127 (2017), 118-129.

DOI: https://doi.org/10.1016/j.ijmecsci.2016.12.022 
[13] M. Safi, L. Baudouin and A. Seuret, Refined exponential stability analysis of a coupled system, IFAC-PapersOnLine 50(1) (2017), 11972-11977.

DOI: https://doi.org/10.1016/j.ifacol.2017.08.1758

[14] Y. Guo, S. Liu and X. H. Ding, The existence of periodic solutions for coupled Rayleigh system, Neurocomputing 191 (2016), 398-408.

DOI: https://doi.org/10.1016/j.neucom.2016.01.039

[15] D. Kampert and U. Epple, Challenges in the modelling and operation of physically coupled systems of systems, IFAC-PapersOnLine 48(1) (2015), 916-917.

DOI: https://doi.org/10.1016/j.ifacol.2015.05.104

[16] K. Gopalsamy, Stability and Oscillations in Delay Differential Equations of Population Dynamics, Kluwer Academic, Boston, 1992. 